\title{
Zur Ultrastruktur der seitlichen Sinnesorgane am Augenhügel von Anoplodactylus pygmaeus (Pycnogonida)
}

\author{
S. Richter \\ Institut für Allgemeine Zoologie der Freien Universität Berlin; Königin-Luise-Str. \\ 1-3, D-1000 Berlin 33
}

\begin{abstract}
Ultrastructure of the lateral sense organs on the ocular tubercle of Anoplodactylus pygmaeus (Pycnogonida). Former light microscopic studies on the lateral sense organs of sea spiders yielded divergent results. Consequently, different authors ascribed different functions to these organs. The present ultrastructural study shows that each lateral sense organ of A. pygmaeus consists of approximately 15 sensory cells of two different types, approximately 20 sheath cells with numerous long microvilli, and an outer cuticular covering. Essentially the same elements are characteristic features of arthropod sensilla. There are, however, differences between the sense organs described in this paper and the sense organs of other arthropods. The inner dendritic segments of sensory cells $\mathrm{S}_{1}$ of the A. pygmaeus lateral sense organs are very short, the sensory cilia are invaginated, and the pericarya of the sensory cells contain electron lucent cytoplasmic regions with large granules (glycogen?). In addition, the lateral sense organs of $A$. pygmaeus lack a marked receptor lymph cavity and junctions between the cells. The results of the present ultrastructural study clearly indicate that the lateral sense organs of $A$. pygmaeus are not glands as was postulated for other sea spider species by earlier authors. Some investigators hypothesized that the lateral sense organs of other sea spider species were auditory organs or rudimentary eyes. The present results do not support such speculations. Some structural details of the sensory cells of A. pygmaeus resemble those found in chemoreceptive or putative chemoreceptive organs of other arthropods. Accordingly, chemoreceptive or thermoreceptive functions should be taken into consideration for the lateral sense organs of $A$. pygmaeus.
\end{abstract}

\section{EINLEITUNG}

Auf dem Augenhügel der meisten Pycnogonida befinden sich 4 Augen. Zwischen ihnen liegt auf jeder Lateralseite des Augenhügels ein Organ, das von Dohrn (1881) entdeckt und "seitliches Sinnesorgan" genannt wurde. Der Bau der seitlichen Sinnesorgane wurde mehrfach mit dem Lichtmikroskop untersucht, von den verschiedenen Untersuchern jedoch recht unterschiedlich beschrieben. Als Folge davon gingen auch die Meinungen hinsichtlich der Funktion dieser Organe weit auseinander. Dohrn (1881) war die Funktion nicht klar, er dachte jedoch an eine eventuelle Gehörfunktion. Loman (1907) hielt die Möglichkeit, daß die fraglichen Organe Sinneswerkzeuge seien, nicht für ausgeschlossen, glaubte aber, daß es sich dabei auch um Drüsen handeln könnte. Sokolow (1911) war ebenfalls dieser Meinung, bemerkt jedoch, daß der Mangel eines Ausführungsganges der Erklärung gewisse Hindernisse in den Weg stellt. Die eingehendste lichtmikroskopische Untersuchung stammt von Wirén (1918). Er sah die Organe 
als mehr oder weniger rudimentäre Augen an, und Hanström (1919, zitiert nach Helfer \& Schlottke, 1935) betrachtete sie als ein Homologon der Frontalorgane von Crustaceen.

Die Widersprüche hinsichtlich des Baues regten dazu an, die Ultrastruktur der Organe bei Anopladactylus pygmaeus Hodge zu untersuchen.

\section{MATERIAL UND METHODE}

Die Tiere wurden an der Küste von Helgoland in einer Tiefe von $4-5 \mathrm{~m}$ bei Hochwasser bzw. 2-3 $\mathrm{m}$ bei Niedrigwasser auf verschiedenen Algenarten gefunden und zusammen mit den Algen und deren Bewuchs nach Berlin gebracht, wo sie im Aquarium bis zu 20 Monaten am Leben blieben.

Die Fixierung der zur Untersuchung im Durchstrahlungselektronenmikroskop bestimmten Individuen wurde nach zahlreichen Variationen vorgenommen mit: $5 \%$ Glutaraldehyd in Cacodylatpuffer, $\mathrm{pH} \mathrm{7,4} \mathrm{(2} \mathrm{Std).} \mathrm{Das} \mathrm{Auswaschen} \mathrm{erfolgte} \mathrm{im} \mathrm{gleichen}$ Puffer über Nacht, die Nachfixierung in $2 \% \mathrm{OSO}_{4}(2 \mathrm{Std})$. Alle Lösungen wurden mit einem Gemisch von synthetischem Meerwasser: $\mathrm{H}_{2} \mathrm{O}$ bidest. $=1: 1$ angesetzt. Die Entwässerung erfolgte in Athanol und Propylenoxid, die Einbettung in Araldit. Geschnitten wurde mit einem Diamantmesser am Ultramikrotom OMU 3 der Firma

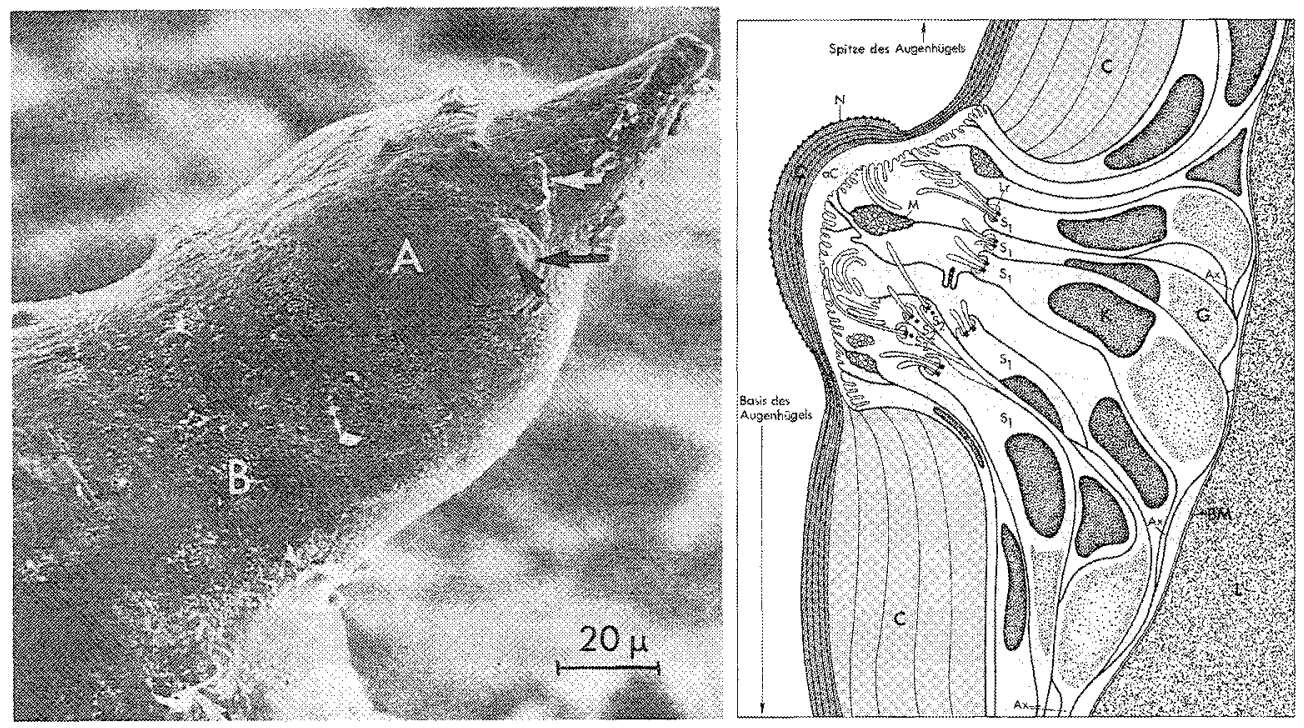

Abb. 1 (links). Rechtes seitliches Sinnesorgan auf dem Augenhügel von Anoplodactylus pygmaeus. A: Auge, B: Bewuchs, D: Mündung einer Hautdrüse, weiße Pfeile: Gabelhaare, schwarzer langer Pfeil: peripherer Bereich des seitlichen Sinnesorgans, schwarzer kurzer Pfeil: zentraler Bereich des seitlichen Sinnesorgans

Abb. 2 (rechts). Schema eines Sagittalschnittes durch ein linkes seitliches Sinnesorgan. Ax: Axone, BM: Basalmembran von Hüllzellen, $C$ : Cuticula, aC: aufgelockerter Bereich der Cuticula, G-helle Punktierung: Granula im elektronentransparenten Bereich der Sinneszellen, K - dunkle Punktierung: Zellkerne, L: Hämolymphe, Lr: Liquorraum, N: "Nippel", $S_{1}$ : Sinneszellen mit je 2 Cilien (Anschnitte), $\mathrm{S}_{2}$ : Sinneszellen mit je 1 Cilium (Anschnitt), übrige Zellen: Hüllzellen mit angeschnit tenen Mikrovilli, M: Querschnitte durch Mikrovilli von Hüllzellen 
Reichert. Die Nachkontrastierung erfolgte mit Uranylacetat und Bleicitrat nach Reynolds (1963). Für die Aufnahmen stand ein Zeiss EM 9 S-2 zur Verfügung.

Die zur Betrachtung im Rasterelektronenmikroskop vorgesehenen Tiere wurden in $70 \%$ Äthanol fixiert, entwässert, mit Gold bedampft und in einem Cambridge-Stereoscan Mark II untersucht.

Ausgewertet wurden nur Aufnahmen der seitlichen Sinnesorgane geschlechtsreifer Weibchen von Anoplodactylus pygmaeus.

\section{ERGEBNISSE}

Auf der Oberfläche des Augenhügels ist die cuticulare Bedeckung der seitlichen Sinnesorgane sichtbar (Abb. 1). Sie hat einen etwa kreisförmigen Umriß, einen Durchmesser von ca. $18 \mu \mathrm{m}$, und ihr oberer Rand befindet sich etwas distal vom oberen Rand der Cornea der beiden benachbarten lateralen Augen. Wie es schon von Dohrn (1881) für einige Pycnogonidenarten beschrieben wurde, ist auch die Cuticula, von der die seitlichen Sinnesorgane von Anoplodactylus pygmaeus bedeckt werden, etwas nach außen vorgewölbt, der ringförmige periphere Bereich weniger stark als der zentrale.

Auf der Oberfläche der cuticularen Bedeckung befinden sich kleine Erhebungen (Abb. 2, 3), die den Nippeln auf der Cornea der Facettenaugen von Insekten ähnlich sehen. Längsschnitte durch den Augenhügel zeigen, daß die Dicke der cuticularen Bedeckung der seitlichen Sinnesorgane nur etwa ein Viertel der Dicke der Cuticula des Augenhügels beträgt. Die inneren Lamellen der cuticularen Bedeckung haben eine andere Beschaffenheit als die äußeren. Die inneren Lamellen sind stark aufgelockert, am stärksten im zentralen Bereich (Abb. 2, 3). Da die Auflockerung bei allen untersuchten Tieren vorhanden war, ist anzunehmen, daß es sich dabei nicht um einen Artefakt handelt.

Unterhalb der cuticularen Bedeckung befinden sich ca. 35 Zellen. Aus Abbildung 2, auf der ein Sagittalschnitt durch ein seitliches Sinnesorgan schematisch dargestellt ist, geht hervor, daß es sich bei diesen Zellen um Sinneszellen und Hüllzellen handelt.

Ein Typ von Sinneszellen $\left(S_{1}\right.$, Abb. 2,4$)$ zeichnet sich vor allem durch sehr große Perikaryen aus, in deren basalem Abschnitt sich ein auffallend großer, nicht von einer Membran begrenzter elektronentransparenter Bereich befindet, der viele elektronendichte Granula enthält. Distal von diesem Bereich liegt der Zellkern, und apikal schließt sich ein kurzer schmalerer Fortsatz an, der vom distalen Ende her eingefaltet ist (Abb. 4, Inset). Am Grunde der Einfaltung entspringen nebeneinander 2 modifizierte Cilien. Jedes Cilium besitzt einen Basalkörper; eine Cilienwurzel war nicht deutlich zu sehen. Vom Basalkörper bis zu der Stelle, wo die Cilien die Einfaltung verlassen, sind im Querschnitt 9 Paar periphere Mikrotubuli vorhanden, zentrale Mikrotubuli fehlen. Wo zentrale Mikrotubuli zu erwarten gewesen wären, enthält das Cilium eine im Längsschnitt fadenförmige dunkle Struktur. Distal von der $9 \times 2$-Struktur sind in den Cilien keine Mikrotubuli zu erkennen. Man findet dort unregelmäßige dunkle Strukturen. Die Sinnescilien enden nahe der Cuticula, die das Sinnesorgan bedeckt. Bei einer Reihe von Tieren zeigten die distalen Abschnitte der Cilien elektronentransparente Anschwellungen (Abb. 5), die um so größer waren, je weniger befriedigend die Fixierung ausfiel.

Das Cytoplasma des Perikaryons sieht bei verschiedenen Tieren unterschiedlich aus. Es gibt Individuen, bei denen das Cytoplasma der Sinneszellen hell erscheint, bei 


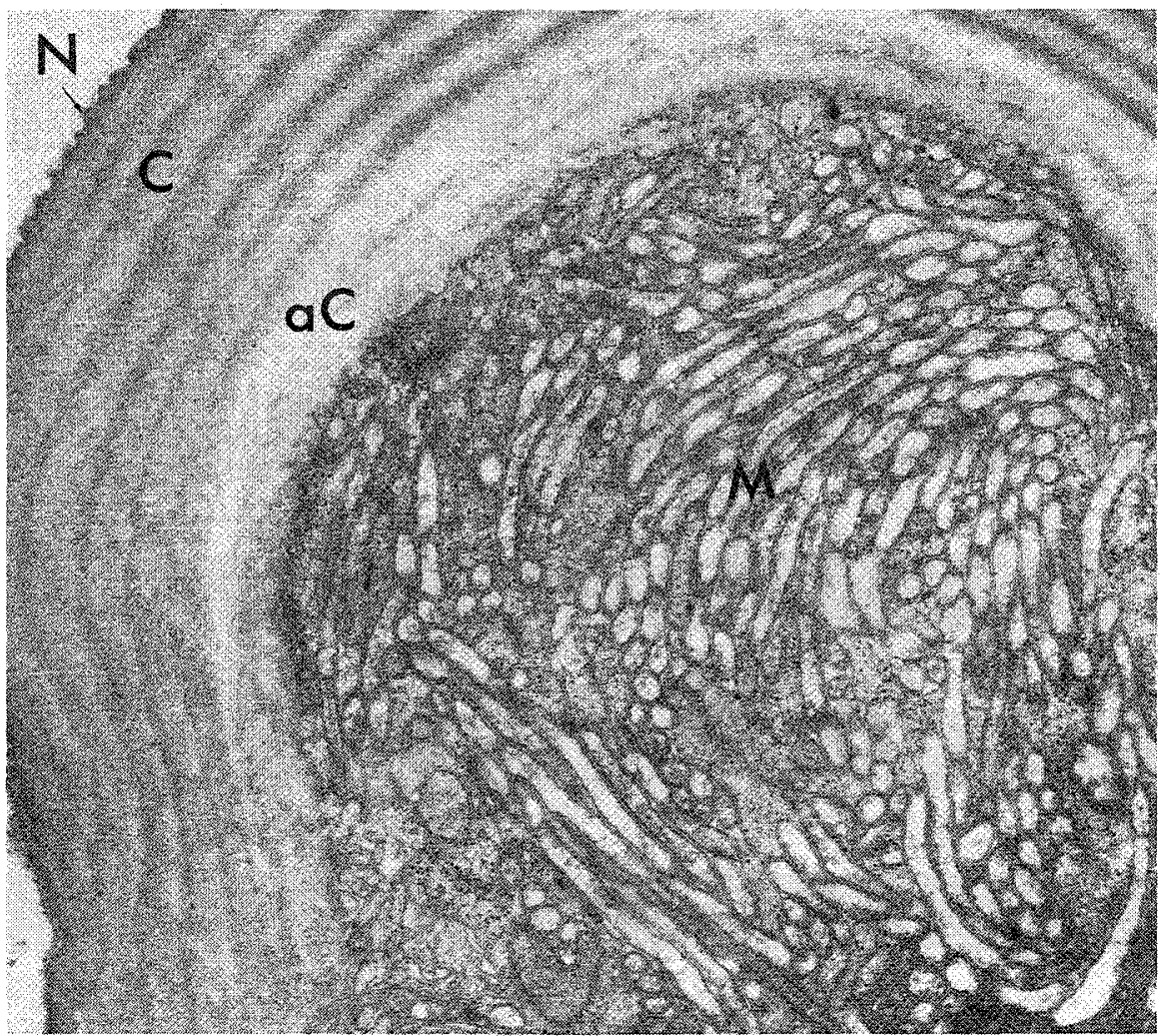

Abb. 3. Tangentialschnitt durch die Mikrovilliregion der Hüllzellen. C: Cuticula, aC: aufgelockerter Bereich der Cuticula, M: Mikrovilli, N: "Nippel", 23750 x

anderen ist das Cytoplasma einzelner (Abb. 6), mehrerer oder fast aller Sinneszellen $(\mathrm{Abb} .4)$ sehr elektronendicht. An Zellorganellen enthalten sowohl die hellen als auch die dunklen Sinneszellen außer dem bereits erwähnten, großen basalen, mit Granula erfüllten Bereich vor allem Mitochondrien. Ihre Anzahl ist in dunklen Sinneszellen in der Regel größer als in hellen. Auch mit unterschiedlichem Material mehr oder weniger stark angefüllte helle Vakuolen, kleine Vesikel, rauhes endoplasmatisches Reticulum, Golgiapparate sowie relativ große elektronendichte Einschlußkörper mit unterschiedlicher Struktur sind in den Perikaryen vorhanden. Die Anzahl der Einschlußkörper ist

Abb. 4 (links). Längsschnitt durch ein seitliches Sinnesorgan. Ax: Axon, B: Bewuchs, C: Cuticula, aC: aufgelockerter Bereich der Cuticula, G: Granula, H: Hüllzellen, K: Zellkerne, L: Hämolymphe, Lz: Hämolymphzelle, M: Mikrovilli der Hüllzellen, $\mathrm{S}_{1}$ : Sinneszellen mit 2 Cilien, 9870 x. Inset: Apikaler Bereich einer Sinneszelle $S_{1}$ (nur 1 Cilium angeschnitten). Ci: Cilium, dis: inneres Segment des Dendriten, H: Hüllzelle, Lr: Liquorraum, Mi: Mitochondrien, 23750 x

Abb. 5 (rechts oben). Cilienanschwellung bei unbefriedigender Fixierung. AC: Cilienanschwellung, $\mathrm{Ci}$ : Cilium, H: Hüllzelle, $\mathrm{S}_{1}$ : Sinneszellen mit 2 Cilien, $11750 \mathrm{x}$

Abb. 6 (rechts unten). Helle und dunkle Sinneszelle $S_{1}$. Ci: Cilium, E: Einschlußkörper, G: Granula, H: Hüllzelle, K: Zellkern, Lr: Liquorraum, M: Mikrovilli, $11750 \mathrm{x}$ 

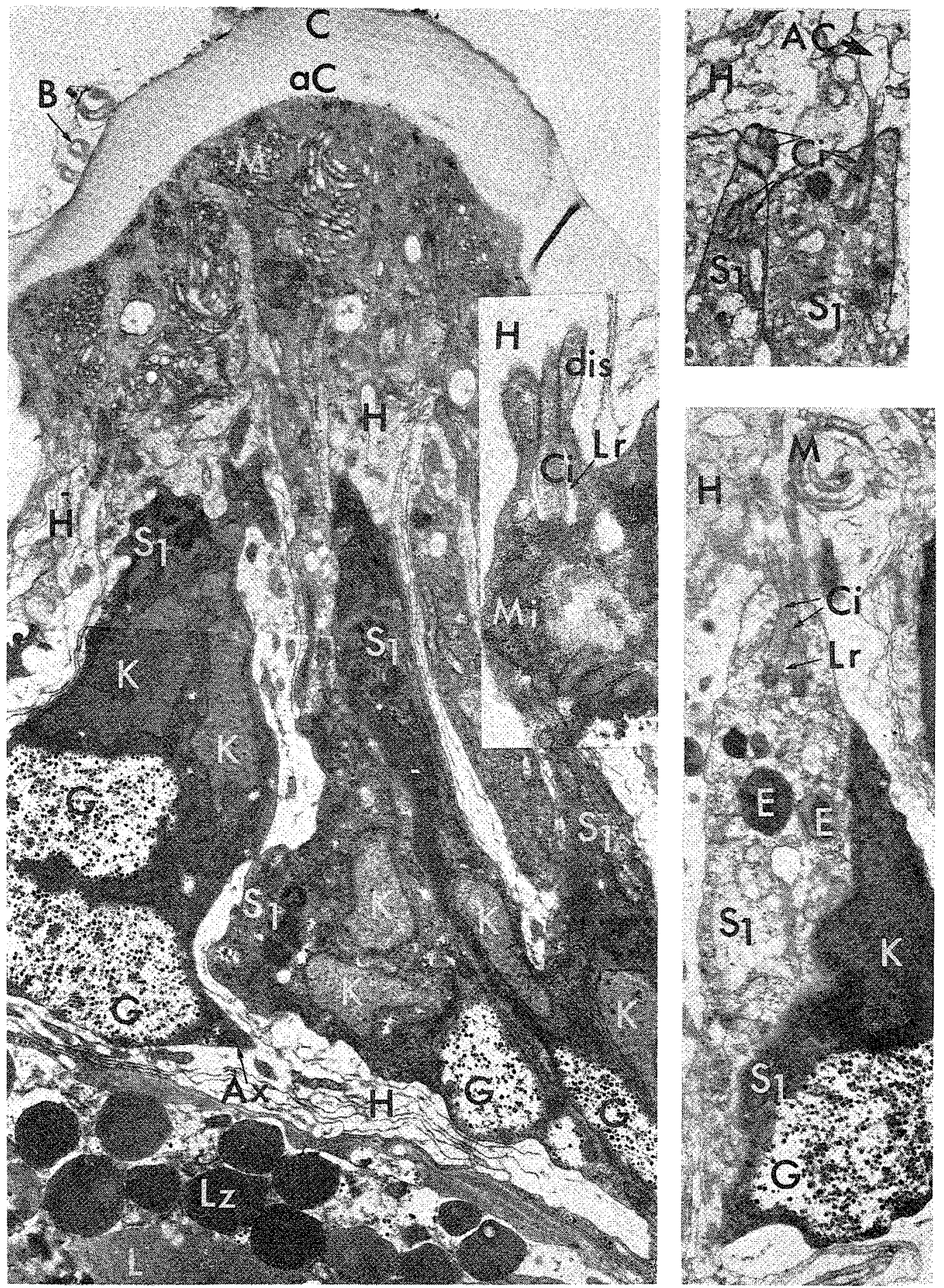
zwar nicht in allen, aber doch in vielen dunklen Sinneszellen größer als in den hellen. Im. Cytoplasma der dunklen Sinneszellen befinden sich außerdem auch distal vom Zellkern kleinere helle, Granula enthaltende Bereiche, die ebenso aussehen wie die erwähnten großen basalen (Abb. 7).

Díe Axone derjenigen Sinneszellen, die sich in der distalen Hälfte des Sinnesorgans befinden, biegen beinahe im rechten Winkel zur Längsachse der Sinneszelle ab (Abb. 2, 8) und verlaufen dann relativ nahe der Cuticula weiter in Richtung zur Basis des Augenhügels. Von Sinneszellen, die in der proximalen Hälfte des Sinnesorgans liegen, gehen die Axone etwa in Richtung der Längsachse der Perikaryen ab (Abb. 2). Die Axone sind dünn - ihr Durchmesser beträgt nur 0,1-0,2 $\mu \mathrm{m}$, und ihr Cytoplasma ist sehr elektronendicht. Zuweilen enthalten die Anfangsabschnitte einiger Axone außer kleinen Mitochondrien auch helle Bereiche, in denen sich dunkle Granula befinden, die den großen, mit dunklen Granula erfüllten Bereichen gleichen, die im basalen Abschnitt der Perikaryen vorhanden sind. Mikrotubuli waren jedoch in den Axonen nie zu erkennen.

Jedes Sinnesorgan enthält mindestens 8 Sinneszellen, von denen jede zwei Sinnescilien besitzt.
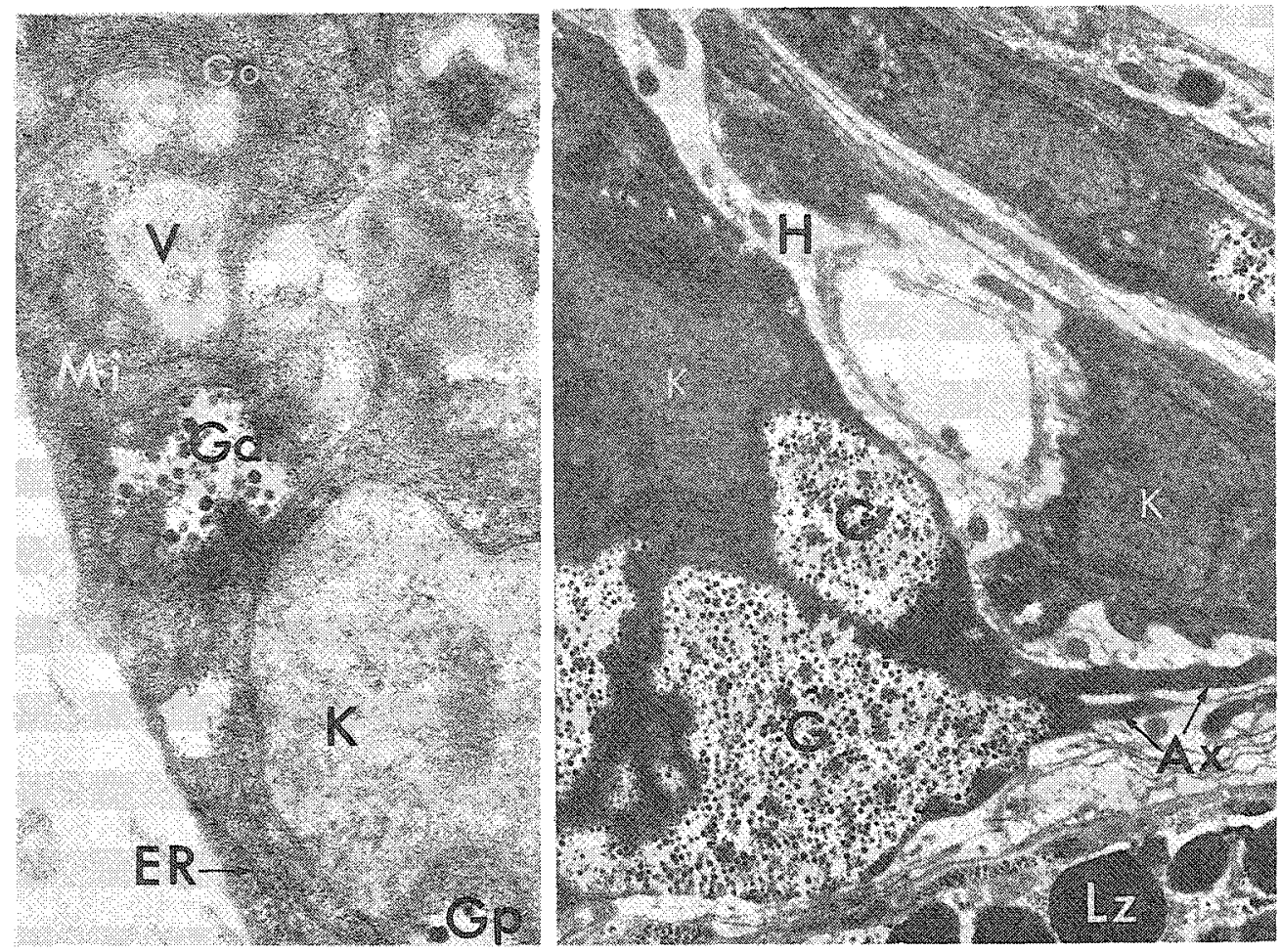

Abb, 7 (links). Perikaryon einer dunklen Sinneszelle $S_{1}$, ER: endoplasmatisches Reticulum, Gd: Gramula distal vom Zellkern, Gp: Gramula proximal vorn Zellkern, Go: Golgiapparat, K: Zellkern, Mi: Mitochondrien, V: Vakuole, 23750 *

Abb. 8 (rechts). Axone von Sinneszellen $S_{1}$. Ax: Axon, G: Granula, H: Hüllzelle, K: Zellkern, Lz: Hämolymphzelle, $11750 \mathrm{x}$ 

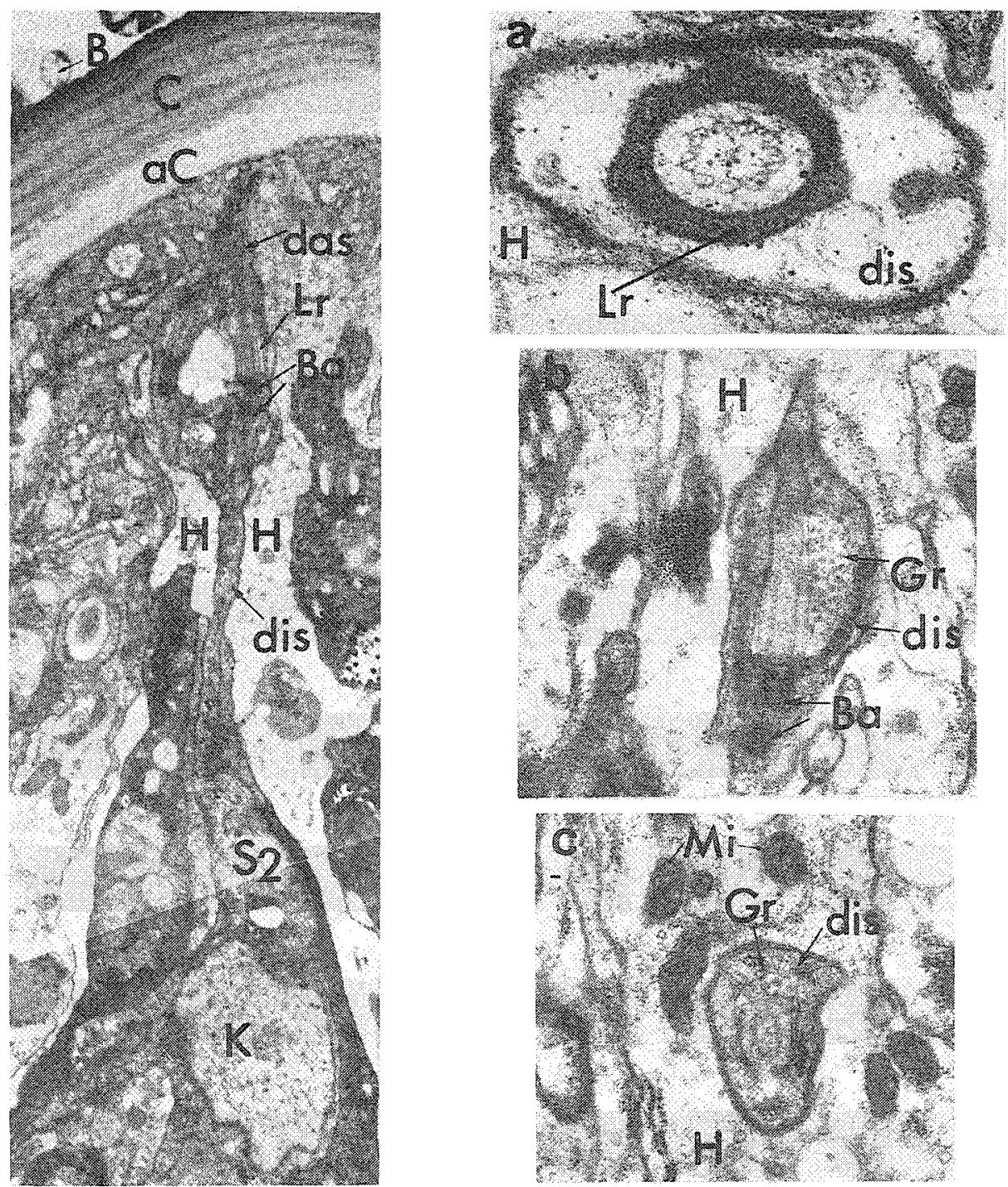

Abb. 9 (links). Sinneszelle $S_{2}$. B: Bewuchs, Ba: Basalkörper, C: Cuticula, aC: aufgelockerter Bereich der Cuticula, das: äuBeres Segment des Dendriten, dis: inneres Segment des Dendriten, H: Hullzelle, K: Zellkern, Lr: Liquorraum, $S_{2}$ : Sinneszelle mit 1 Cilium, $11750 \times$

Abb. 10 (rechts). (a) Querschnitt durch das eingesenkte Cilium einer Sinneszelle $S_{2}$. dis: inneres Segment des Dendriten, H: Hullzelle, Lr: Liquorraum, $70000 \mathrm{x}$. (b) Gramula im Liquorraum einer Sinneszelle $S_{2}$, Ba: Basalkörper, dis: inneres Segment des Dendriten, Gr: Granula, H: Hülzelle, 23750 x. (c) Querschnitt durch die apikale Einfaltung des dendritischen Fortsatzes einer Sinneszelle $\mathrm{S}_{2}$. dis: inneres Segment des Dendriten, Gr: Granula, H: Hüllzelle, Mi: Mitochondrien, 
Der zweite Sinneszelltyp $\left(S_{2}\right.$, Abb. 9) gleicht dem ersten hinsichtlich der Struktur des Perikaryons. Der dendritische Fortsatz dieser Sinneszellen besteht jedoch aus einem relativ langen, schlanken inneren und einem äußeren Segment. Das äußere Segment beginnt als ein eingesenktes, modifiziertes Cilium $_{r}$ das zwei hintereinander gelegene Basalkörper besitzt. Es liegt ein wenig distal von den Cilienstrukturen der Sinneszellen $S_{1}$. Eine Cilienwurzel war auch hier nicht deutlich zu erkennen. Wie der Querschnitt durch das Cilium (Abb. 10a) zeigt, enthält es, ebenso wie die beiden Cilien der Sinneszellen $\mathrm{S}_{1}, 9$ Paar periphere Mikrotubuli. Im Zentrum befindet sich eine graue Struktur. Die Mikrotubuli der $9 \times 2$-Struktur sind kurz, sie enden etwa an der Stelle, an der das äußere Segment des Dendriten die Einfaltung verläßt. Die Struktur des distalen Bereichs des äußeren Segmentes gleicht dem entsprechenden Abschnitt der Sinneszellen $\mathrm{S}_{1}$. Das dendritische Außensegment endet dicht unterhalb der Cuticula.

Obwohl ich die Axone nicht mit Sicherheit identifizieren konnte, bin ich der Meinung, daß es sich auch bei den $\mathrm{S}_{2}$-Zellen um Sinneszellen handelt. Wie Querschnitte durch den Bereich des Sinnesorgans zeigen, in dem sich die Cilienstruktur des dendritischen Fortsatzes befindet, sind mindestens 7 Sinneszellen vom Typ $\mathrm{S}_{2}$ in einem Sinnesorgan vorhanden. Drei dieser 7 Sinneszellen stehen dicht nebeneinander.

Bei einem Weibchen zeigten einige Sinneszellen vom Typ $S_{2}$ eine Besonderheit, die erwähnt werden soll, weil sie auf eine sekretorische Tätigkeit dieser Sinneszellen hinweist. Im Gegensatz zu den Sinneszellen vom Typ $\mathrm{S}_{2}$ der übrigen untersuchten Individuen ist hier die Einfaltung am distalen Ende des inneren dendritischen Segmentes, an deren Basis das Sinnescilium beginnt, etwas erweitert und mit kleinen Granula erfüllt (Abb. 10b). Derartige Granula bzw. Vesikel gleicher Größe befinden sich auch im Cytoplasma des inneren dendritischen Segments im Bereich der Einfaltung. Auf einem Querschnitt durch die apikale Einfaltung des dendritischen Fortsatzes einer Sinneszelle ist ein Granulum an der Grenze zwischen dem Cytoplasma und dem Lumen der Einfaltung sichtbar (Abb. 10c). Weiter distal sind ebenso aussehende Granula bzw. Vesikel sowohl im Interzellularraum zwischen den Mikrovilli der noch zu beschreibenden Hüllzellen als auch im Inneren von Mikrovilli vorhanden. Schließlich befinden sich Granula auch extrazellulär an der Grenze zwischen den Mikrovilli und der stark aufgelockerten cuticularen Bedeckung des Sinnesorgans (Abb. 11).

Die ca. 20 Hüllzellen, die zu einem Sinnesorgan gehören, tragen an ihrem distalen Ende eine große Anzahl langer Mikrovilli, die unregelmäßig verlaufen. Einige Mikrovilli scheinen verzweigt $\mathrm{zu}$ sein $(\mathrm{Abb}, 11)$. Auf Schnitten, die dicht unterhalb der Cuticula verlaufen, erkennt man vorwiegend Anschnitte der Mikrovilli (Abb. 3). Da einerseits die Mikrovilli etwa den gleichen Durchmesser besitzen wie die distalen Abschnitte der Sinnescilien und andererseits im Inneren der Mikrovilli ebenso wie in den distalen Abschnitten der Sinnescilien elektronentransparentere Abschnitte mit solchen abwechseln können, die elektronendichteres Material enthalten, sind die distalen Enden der Sinnescilien von den Mikrovilli nicht mit Sicherheit zu unterscheiden.

Auf Längsschnitten durch das Sinnesorgan (Abb. 4) ist zu sehen, daß sich einige Hüllzellen zwischen den Sinneszellen befinden, andere dagegen an der Peripherie des Sinnesorgans. Unterhalb der Mikrovilliregion bilden peripher gelegene, abgeflachte Hüllzellen einen geschlossenen Ring. In dem Bereich, in dem sich die Cilienregion der Sinneszellen befindet, werden die dendritischen Fortsätze und auch die apikalen Enden der Perikaryen der Sinneszellen vollständig von einer oder zwei Hüllzellen umgeben. 


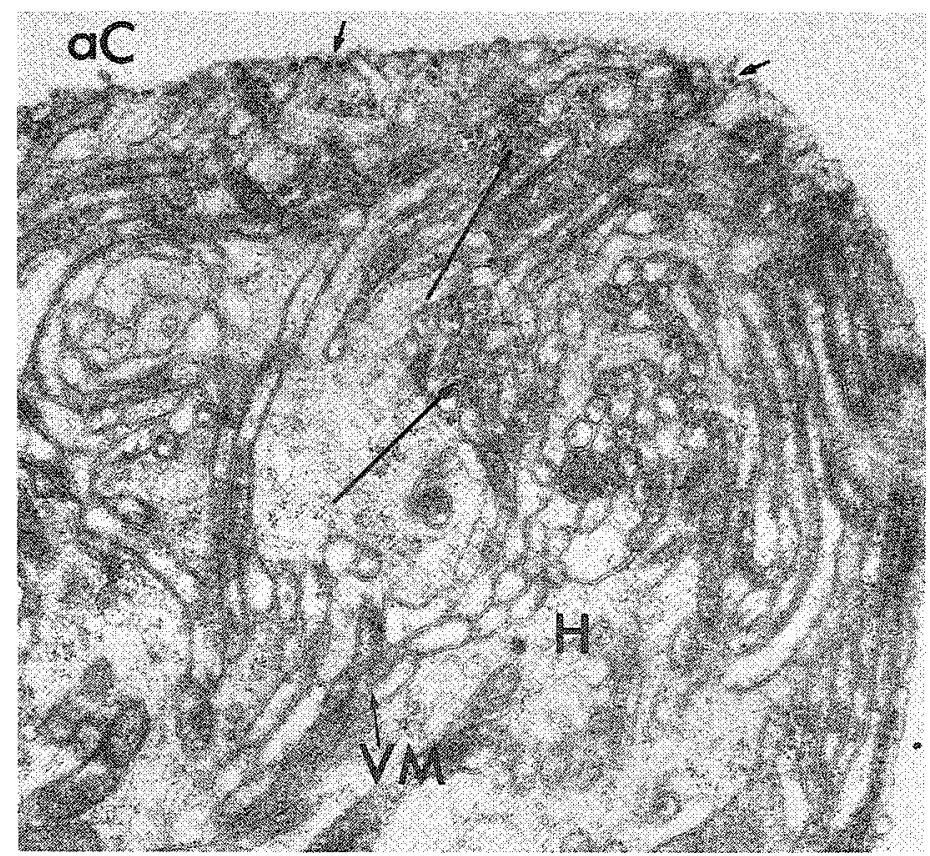

Abb. 11. Schnitt durch die Mikrovilliregion von Hüllzellen. aC: aufgelockerter Bereich der Cuticula, Pfeile: Granula, H: Hüllzelle, VM: Verzweigung eines Mikrovillus, $23750 \times$

Weiter basal sind die Verhältnisse komplizierter. Hier werden die Perikaryen mehrereI Sinneszellen gemeinsam von mehr als zwei Hüllzellen umschlossen.

Die Hüllzellen besitzen einen großen Zellkern, unter dem sich meistens ein Golgiapparat befindet. Das Cytoplasma der Hüllzellen ist vor allem in der proximalen Zellhälfte wenig elektronendicht. An Zellorganellen kommen in diesem Bereich nur einzelne kleine Mitochondrien und wenig rauhes endoplasmatisches Reticulum vor. In der distalen Zellhälfte nimmt vor allem bei denjenigen Hullzellen, die sich zwischen den Sinneszellen befinden, die Anzahl der Mitochondrien zu. Das Cytoplasma enthält außerdem neben einem schwach ausgebildeten endoplasmatischen Reticulum kleine Vesikel und Vakuolen verschiedener Größe mit wahrscheinlich unterschiedlichem Inhalt.

Trotz weitgehend guter Strukturerhaltung war bei mehreren Tieren im Bereich der Mikrovilli das Cytoplasma einzelner Hüllzellen degeneriert bis völlig aufgelöst (Abb. 12). Aufgrund welcher Ursachen es zu dieser lokal begrenzten Degeneration gekommen sein könnte, ist nicht klar erkennbar.

Von der Leibeshöhlenflüsigkeit werden die seitlichen Sinnesorgane durch die Basis von Hüllzellen bzw. deren Basalmembranen abgegrenzt. 


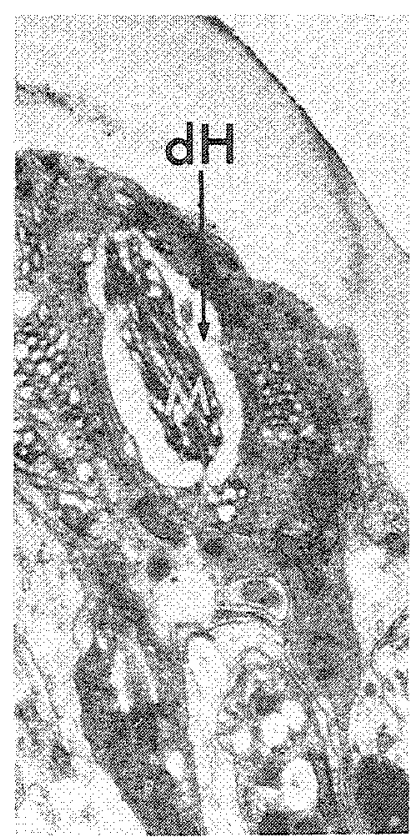

Abb. 12. Lokale Degeneration im Bereich der Mikrovilliregion von Hüllzellen. dH: degenerierte Hüllzellen, M: Mikrovilli, 11750 x

\section{DISKUSSION}

Die seitlichen Sinnesorgane von Anoplodactylus pygmaeus enthalten die gleichen Bausteine wie die Sensillen von Arthropoden: Sinneszellen, Hüllzellen, cuticularer Apparat. Es ist anzunehmen, daß der Bau der seitlichen Sinnesorgane bei verschiedenen Pycnogoniden-Arten, die einen unterschiedlichen Habitus besitzen, in unterschiedlichen Tiefen leben bzw. eine unterschiedliche Lebensweise führen, variiert. Ob die Variabilität jedoch so weit geht, daß sich am Augenhügel einiger Arten seitliche Sinnesorgane und an entsprechender Stelle bei anderen Arten Drüsen befinden letzteres hielt nach lichtmikroskopischen Untersuchungen Sokolow (1911) für fünf Pycnogonidenarten und Loman (1907) für Phoxichilidium femoratum für wahrscheinlich -, muß durch die Neuuntersuchung dieser Arten mit dem Elektronenmikroskop geklärt werden.

Bei Arthropoden scheint es selten vorzukommen, daß ein und dasselbe Sinnesorgan zwei morphologisch unterschiedliche Typen von Sinneszellen enthält, wie dies bei den seitlichen Sinnesorganen unter der Voraussetzung der Fall ist, daß es sich auch bei den Zellen $\mathrm{S}_{2}$ um Sinneszellen handelt. Meines Wissens ist dies bisher nur bei dem multifunktionellen Sinneskomplex an der Antennenspitze des Collembolen Allacma fusca gefunden worden (Altner \& Thies, 1978).

Sinneszellen mit zwei dendritischen Außensegmenten besitzt eine Reihe von Arthropoden (Haupt, 1973, Schönrock, 1981). Die Sinneszellen $S_{1}$ von Anoplodactylus pygmaeus unterscheiden sich jedoch von diesen Sinneszellen zum einen dadurch, daß ihr dendritisches Innensegment sehr kurz ist; nur bei günstiger Schnittführung (Abb. 4, 
Inset) kann man es erkennen, und zum anderen sind die Cilien eingesenkt. Eingesenkte Sinnescilien sind mir in Sinnesorganen anderer Arthropoden nicht bekannt. Einzeln stehende epitheliale Sinneszellen von Coelenteraten, Turbellarien, Nemertinen, Oligochaeten und am Saugnapf von Octopus besitzen dagegen eingesenkte Sinnescilien. Für diese epithelialen Sinneszellen wird eine chemorezeptorische Funktion angenommen (Altner \& Prillinger, 1980). Anschwellungen an den Sinnescilien (Abb. 5) gibt es bei verschiedenen Tieren. Bei olfaktorischen Sinnesorganen an den Antennulae von Pachygrapsus crassipes waren Anschwellungen sowohl nach chemischer als auch nach physikalischer (freeze-substitution) Fixierung (Frisch \& Everingham, 1971) vorhanden. Nach diesen Autoren repräsentieren Cilienanschwellungen Orte, an denen ein Permeabilitätswechsel erfolgt, vielleicht als Antwort auf Stimulation durch Geruchsmoleküle.

Die Tatsache, daß bei den seitlichen Sinnesorganen weder in den Axonen der Sinneszellen noch in deren distalen dendritischen Segmenten Mikrotubuli zu erkennen sind, ist auf einen Artefakt zurückzuführen. Auch in den Axonen der Retinazellen und in den Axonen und dendritischen Fortsätzen derjenigen Sinneszellen, von denen Haare am Augenhügel innerviert werden, waren keine Mikrotubuli zu finden. Nur in Querschnitten durch den Tubularkörper von wahrscheinlich mechanorezeptorischen Sinneszellen sind Mikrotubuli sichtbar.

Ungewöhnlich für Sinneszellen von Arthropoden sind die großen, hellen, mit Granula erfüllten Bereiche im Perikaryon. Gleich aussehende Granula befinden sich auch in allen Retinazellen und in Sinneszellen, die zu Sinneshaaren gehören. Bei Tieren, die vor der Fixierung nur kurze Zeit im Aquarium gelebt hatten, enthalten auch Epidermiszellen und einige Hämolymphzellen derartige Granula. Wenn die Tiere vor der Fixierung lange Zeit unter sicherlich nicht optimalen Bedingungen im Aquarium gelebt hatten, waren die Granula aus den Epidermis- und Hämolymphzellen verschwunden, nicht jedoch aus den Sinneszellen. In letzteren nahm nur die Anzahl der Granula ab, und es traten Vakuolen innerhalb der Granula auf. Aus welchem Material die Granula bestehen, muß durch eine histochemische Untersuchung geklärt werden. Aufgrund des optischen Eindrucks könnte man an Glycogen $(\alpha+\beta)$ denken. Bei wirbellosen Tieren ist Glycogen entweder ein Zeichen intensiver Stoffwechselaktivität des betreffenden Organs oder es dient als Reservestoff (Gaill, 1980). Die Verminderung der Anzahl der Granula in den Sinneszellen und ihr völliges Verschwinden aus den übrigen Zellen deutet auf einen Abbau hin.

Auf welchen Ursachen die unterschiedliche Kontrastierung der Sinneszellen bei verschiedenen Tieren beruht, ist unklar. Der Kontrastunterschied kann durch Fixierung und Kontrastierung verstärkt, jedoch nicht allein ausgelöst worden sein, da bei ein und demselben Tier helle und dunkle Sinneszellen gleichzeitig vorhanden sind. Außerdem trat bei den Hüllzellen nie ein Kontrastunterschied auf. Es wäre denkbar, daß die unterschiedliche Kontrastierung der Sinneszellen einen unterschiedlichen physiologischen Zustand der Tiere bzw. einen unterschiedlichen Funktionszustand der Sinneszellen anzeigt. Die größere Anzahl von Mitochondrien und Einschlußkörpern in den dunklen Sinneszellen deutet möglicherweise auf eine größere Aktivität dieser Zellen gegenüber den hellen Sinneszellen hin. Auf einen besonderen Funktionszustand weisen auch die Granula hin, die nur bei einem einzigen Tier vorhanden waren und innerhalb der Einfaltung am distalen Ende des inneren dendritischen Segments der Sinneszellen $\mathrm{S}_{2}$ besonders zahlreich auftraten (Abb. 10b). 
Erstaunlich ist bei einem Vergleich mit epidermalen Sinnesorganen anderer Arthropoden, daß die dendritischen Fortsätze der Sinneszellen der seitlichen Sinnesorgane nur an der Basis des distalen dendritischen Segmentes, soweit dieses in das proximale Segment eingesenkt ist, von einem winzigen Liquorraum umgeben werden. Es scheinen außerdem Membrankontaktstrukturen völlig zu fehlen. Auch die von Green (1981) zwischeri Darmepithelzellen von Ammothea clausi gefundene besondere Form von Membrankontaktstrukturen war in den seitlichen Sinnesorganen von Anoplodactylus pygmaeus nicht zu finden. Fehlende Membrankontakte zwischen den Dendriten erwähnt auch Heimann (1979) für die Sinneszellen in den Sinnesschläuchen von Conchoecia spinirostris.

Aufschlüsse über Funktionen der Hüllzellen sind von einer Untersuchung der Ontogenie der seitlichen Sinnesorgane zu erwarten. Nur durch eine solche Untersuchung kann auch geklärt werden, ob die seitlichen Sinnesorgane einfachen oder komplexen Sensillen anderer Arthropoden entsprechen.

Querschnitte durch die Mikrovilliregion der Hüllzellen (Abb. 3) könnten den Eindruck hervorrufen, daß diese Zellen Photorezeptoren seien. Die seitlichen Sinnesorgane sind zwar keine rückgebildeten Augen mit prinzipiell gleichem Bau wie die normalen Augen (Wirén, 1918), sie könnten aber extraoculare Photorezeptoren enthalten, wie sie z. B. bei Polychaeten (Yoshida, 1979) gefunden worden sind. Auch Mollusken (Mauro, 1977) besitzen derartige Sinnesorgane. Dieser Eindruck wird verstärkt durch die Ausbildung von "Nippeln" auf der Cuticula und dadurch, daß sich die Pigmentschicht von zwei benachbarten Augen dicht unter der Basis der seitlichen Sinnesorgane befindet. Bei Cephalopoda (Decapoda) spielen extraoculare Photorezeptoren wahrscheinlich eine wichtige Rolle bei der Regulation circadianer oder jahreszeitlicher Aktivitäten, u. a. beim Fortpflanzungsverhalten (Mauro, 1977). Auch bei PYcnogonum litorale wird die normale winterliche Fortpflanzungsperiode von der Photoperiode beeinflußt (Schmidt \& Bückmann, 1971). Es wäre daher vorstellbar, daß extraoculare Photorezeptoren auch bei Pycnogoniden zyklische Vorgänge steuern.

Wenn die seitlichen Sinnesorgane tatsächlich extraoculare Photorezeptoren enthielten, so wären diese in den mit vielen langen Mikrovilli versehenen Zellen zu suchen. Als Sinneszellen müßten diese Zellen jedoch Axone besitzen. Da ich Axone nicht finden konnte, handelt es sich bei den fraglichen Zellen sicherlich um Hüllzellen. Damit ist von seiten der Feinstruktur her kein Hinweis auf eine mögliche Funktion der mit Mikrovilli versehenen Zellen als extraoculare Photorezeptoren gegeben.

Ob die seitlichen Sinnesorgane der Pycnogonida ein Homologon der Frontalorgane der Crustaceen sind (Hanström, 1919) bezweifelte schon Schlottke (1935). Er war der Meinung, daß die von Lang (1905) beschriebenen Organe einiger Hydracarinen den seitlichen Sinnesorganen der Pycnogoniden hinsichtlich ihrer Lage, Innervierung und rätselhaften Funktion - sie wurden als rudimentäre Augen oder chemische Sinnesorgane angesehen sowie für andere Funktionen in Anspruch genommen - viel ähnlicher seien als die Frontalorgane der Crustaceen. Die Frage der Homologie kann aufgrund der Untersuchung der Feinstruktur der seitlichen Sinnesorgane von Anoplodactylus pygmaeus nicht diskutiert werden, um so weniger, als sich inzwischen herausgestellt hat, daß es innerhalb der Crustaceen vier verschiedene Typen von Frontalorganen gibt, die nicht einmal einander homolog sind (Eloffson, 1966).

Welche Funktion die seitlichen Sinnesorgane von Anoplodactylus pygmaeus haben 
könnten, bleibt ungewiß. Im Hinblick auf die oben erwähnten, bei Chemorezeptoren bzw. vermutlichen Chemorezeptoren anderer Arthropoden ebenfalls vorhandenen Merkmale der Sinneszellen dürfte die Untersuchung der seitlichen Sinnesorgane auf eine chemorezeptorische oder auch thermorezeptorische Funktion die beste Aussicht auf Erfolg versprechen. Es ist bekannt, daß stimulierende Substanzen die Cuticula passieren können (Laverack, 1974), und es ist vorstellbar, daß der aufgelockerte Bereich der Cuticula, mit dem nicht nur die Mikrovilli der Hüllzellen, sondern auch die Endabschnitte der dendritischen Fortsätze der Sinneszellen in Kontakt stehen, als Liquorraum funktioniert. Wie Stock (1978) für drei Pycnogonidenarten nachgewiesen hat, können Pycnogoniden die Art der chemischen Substanzen, die von Beutetieren produziert werden, sehr präzis unterscheiden. Wo sich die dafür zuständigen Rezeptoren befinden, ist noch nicht bekannt. Als eine der Haltungsbedingungen für Pycnogonum litorale erwähnen Meyer \& Bückmann (1963), daß das Wasser gekühlt werden muß. Das gleiche trifft für die Haltung von Anoplodactylus pygmaeus zu. Dieser Befund könnte auf das Vorhandensein von Thermorezeptoren bei Pycnogoniden hinweisen.

Danksagung. Mein besonderer Dank gilt der Biologischen Anstalt Helgoland für die mehrfache Zurverfügungstellung eines Gastlabors sowie den Angehörigen der Tauchergruppe dieses Institutes für die Beschaffung von Kratzmaterial. Von den Mitarbeitern des Instituts für Allgemeine Zoologie in Berlin danke ich Herrn Dr. H. M. Borchert für seine Hilfe beim Bestimmen der Tiere, Herrn Prof. Dr. Pfannenstiel für die Übersetzung des Abstracts, Herrn Dr. J. Walther für kritische Durchsicht des Manuskriptes und Herrn Dr. U. Schönrock für anregende Diskussionen während der Entstehung der Arbeit. Frau Ch. Grünig möchte ich herzlich danken für ihre sorgfältige und geschickte technische Assistenz und Herrn P. Adam für die Anfertigung der Schemazeichnung. Für die Aufnahmen am Rasterelektronenmikroskop bedanke ich mich bei Herrn Neubauer (Zentraleinrichtung für Elektronenmikroskopie der TU Berlin).

\section{ZITIERTE LITERATUR}

Altner, H. \& Prillinger, L., 1980. Ultrastructure of invertebrate chemo-, thermo-, and hygroreceptors and its functional significance. - Int. Rev. Cytol. 67, 69-139.

Altner, H. \& Thies, G., 1978. The multifunctional sensory complex in the antennae of Allacma fusca (Insecta). - Zoomorphologie 91, 119-131.

Dohrn, A., 1881. Die Pantopoden des Golfes von Neapel und der angrenzenden Meeres-Abschnitte. - Fauna Flora Golf Neapel 3, 1-252.

Eloffson, R., 1966. The nauplius eye and frontal organs of the non-Malacostraca (Crustacea). Sarsia 25, 1-128.

Frisch, D. \& Everingham, J. W., 1972. Fine structure of crab olfactory cilia: Non-chemical fixation; environmental effects. In: Olfaction and taste. Ed. by D. Schneider, Wiss. Verl. Ges., Stuttgart, 5-12.

Gaill, F., 1980. Glycogen and degeneration in the pyloric gland of Dendrodoa grossularia (Ascidiaceae, Tunicata). - Cell Tiss. Res. 208, 197-206.

Green, Cr., 1981. A variation of the smooth septate junction in sea-spiders Ammothea clausi (Pycnogonida). - Tissue Cell 13, 189-195.

Hanström, B., 1919. Zur Kenntnis des zentralen Nervensystems der Arachnoiden und Pantopoden nebst Schlußfolgerungen betreffs der Phylogenie der genannten Gruppen. Diss., Univ. Lund, $191 \mathrm{pp}$.

Haupt, J., 1973. Die Ultrastruktur des Pseudoculus von Allopauropus (Pauropoda) und die Homologie der Schläfenorgane. - Z. Morph. Ökol. Tiere 76, 173-191.

Heimann, P., 1979. Fine structure of sensory tubes on the antennule of Conchoecia spinirostris (Ostracoda, Crustacea). - Cell Tiss. Res. 202, 461-477.

Helfer, H. \& Schlottke, E., 1935. IV Pantopoda. - Bronn's Kl. Ordn. Tierreichs 5, 1-314. 
Lang, P., 1905. Über den Bau der Hydrachnidenaugen. - Zool. Jb. (Anat. Ontogenie Tiere) 21, 453-494.

Laverack, M. S., 1974. The structure and function of chemoreceptor cells. In: Chemoreception in marine organisms. Ed. by P. T. Grant \& A. M. Mackie. Acad. Press, New York, 1-48.

Loman, J. C. C., 1907. Biologische Beobachtungen an einem Pantopoden. - Tijdschr. ned. dierk. Vereen. 10, 255-284.

Mauro, A., 1977. Extra-ocular photoreceptors in cephalopods. - Symp. zool. Soc. Lond. 38, $287-306$.

Meyer, K.-E. \& Bückmann, D., 1963. Die Häutungen des Pantopoden Pycnogonum litorale (Ström.). - Zool. Anz. (Suppl.) 26, 604-609.

Reynolds, E. S., 1963. The use of lead citrate at high $\mathrm{pH}$ as an electron-opaque stain in electron microscopy. - J. Cell Biol. 17, 208-212.

Schmidt, H.-W. \& Brückmann, D., 1971. Beobachtungen zur Lebensweise von Pycnogonum litorale (Ström.) (Pantopoda). - Oecologia 7, 242-248.

Schönrock, G.-U., 1981. Feinstruktur und Entwicklung der antennalen Sensillen bei Polydesmus coriaceus Porath 1871 (Diplopoda, Polydesmida). Diss., F. U. Berlin, 237 pp.

Sokolow, I., 1911. Über den Bau der Pantopodenaugen. - Z. wiss. Zool. 98, 339-380.

Stock, J. H., 1978. Experiments on food preference and chemical sense in Pycnogonida. - J. Linn. Soc. Zool. 63, 59-74.

Wirén, E., 1918. Zur Morphologie und Phylogenie der Pantopoden. - Zool. Bidr., Upps. 6, 41-181.

Yoshida, M., 1979. Extraocular photoreception. In: Handbook of sensory physiology. Ed. by H. Autrum. Springer, 7, 6A, Berlin, 581-640. 